Case Report

\title{
Paradoxical Vestibular Syndrome Caused by a Presumptive Cerebellar Infarction in a Rabbit
}

\author{
Rafael García, DVMª, Sonia Añor, DVM, PhD, Dip ACVIM (Neurology), Dip ECVN ${ }^{\mathrm{a}, \mathrm{b}, *}$, \\ Cristian de la Fuente, DVM, PhD, Dip ECVN ${ }^{\mathrm{a}, \mathrm{b}}$, Rosa Novellas, DVM, PhD, Dip ECVDI ${ }^{\mathrm{b}, \mathrm{c}}$, \\ Vicente Soler, DVM ${ }^{\mathrm{d}}$, Jaume Martorell, DVM, PhD, Dip ECZM ${ }^{\mathrm{b}, \mathrm{d}}$
}

\author{
Keywords: \\ paradoxical vestibular syndrome \\ rabbit \\ cerebrovascular accident \\ magnetic resonance imaging
}

${ }^{a}$ Servei de Neurologia, Fundació Hospital Clínic Veterinari, Campus Universitat Autònoma de Barcelona, Bellaterra, Spain

${ }^{b}$ Departament de Medicina i Cirurgia Animal, Facultat de Veterinària, Edifici V, Universitat Autònoma de Barcelona, Bellaterra, Spain

${ }^{c}$ Servei de Diagnòstic per la Imatge, Fundació Hospital Clínic Veterinari, Campus Universitat Autònoma de Barcelona, Bellaterra, Spain

${ }^{d}$ Servei d'Exòtics, Fundació Hospital Clínic Veterinari, Campus Universitat Autònoma de Barcelona, Bellaterra, Spain
A 6-year-old, female spayed rabbit (Oryctolagus cuniculus) presented with right paradoxical vestibular signs. Magnetic resonance imaging was performed and findings were consistent with an ischemic infarct of the cerebellum. The patient improved gradually and was free of clinical signs at the time this article was written. To the authors' knowledge this is the first case report of a paradoxical vestibular syndrome in a rabbit secondary to a presumptive ischemic infarct. Strokes should be included in the differential diagnosis of central vestibular syndrome in rabbits.

(C) 2021 The Authors. Published by Elsevier Inc. This is an open access article under the CC BY-NC-ND license (http://creativecommons.org/licenses/by-nc-nd/4.0/)

\section{Introduction}

Central vestibular disease is common in pet rabbits, and head tilt is the most common clinical sign. ${ }^{1}$ Although different etiologies have been reported, meningoencephalitis caused by Encephalitozoon cuniculi (E cuniculi) infection appears to be the most frequent ${ }^{2}$ Other causes are rarely recognized. Several articles describe the effects of artificially induced vascular occlusion of rabbit cerebral vessels ${ }^{3-5}$ but reports of naturally occurring cerebrovascular accidents are lacking in small mammal medicine. To the authors knowledge, this is the first report of a presumptive cerebellar infarct in a rabbit diagnosed by magnetic resonance imaging (MRI).

\section{Case Presentation}

A 6-year-old, female spayed, domestic rabbit (Oryctolagus cuniculus) was presented for an acute onset of rolling to the left. The rabbit's diet consisted mainly of unlimited amounts of hay and daily fruits and vegetables. Pellets were restricted. She was housed in a big cage with solid floor and side wires. Physical examination revealed a good body condition of $3 / 5$, a heart rate of 240 beats per minute (reference range:154-300), a respiratory rate of 200 breaths per minute (reference range: $30-60$ ), and a body temperature of $37.8^{\circ} \mathrm{C}$ (reference

*Corresponding author. Sonia Añor, DVM, PhD, Dip ACVIM (Neurology), Dip ECVN, Departament de Medicina i Cirurgia Animal, Facultat de Veterinària, Edifici V, Universitat Autònoma de Barcelona, Bellaterra 08193, Spain

E-mail address: sonia.anor@uab.cat (S. Añor).

Abbreviations: E. cuniculi, Encephalitozoon cuniculi; MRI, Magnetic resonance imaging; T2W, T2 weighted; T1W, T1 weighted; FLAIR, Fluid attenuated inversion recovery; TR, Repetition time; TE, Echo time; DWI, Diffusion weighted imaging; ADC, Apparent diffusion coefficient; CSF, Cerebrospinal fluid; CNS, Central nervous system range: $38^{\circ}-40^{\circ} \mathrm{C}$ ). Neurological examination showed an alert mental status, a left head tilt, reluctance to walk with left vestibular ataxia, and a tendency to fall to the left side. Postural reactions were severely delayed in the right thoracic and pelvic limbs. Cranial nerve evaluation was normal except for a ventral positional strabismus of the left eye and a bilateral rotary nystagmus with the fast phase to the left. Spinal reflexes were considered normal and there were no signs of spinal hyperesthesia. The remainder of the physical and neurological examinations were unremarkable. The neurological examination was considered consistent with a right central (paradoxical) vestibular syndrome. Initial differential diagnoses included neoplastic, inflammatory/infectious, or vascular diseases.

Blood tests (complete blood cell count, serum biochemistry) were within normal values. Thoracic and abdominal radiographs and abdominal ultrasonography did not show any abnormalities.

A brain MRI was performed using a $0.4 \mathrm{~T}$ permanent magnet unit (Hitachi Aperto Lucent Hitachi Medical Systems, Madrid, Spain). A standardized protocol was used (T2W: TR $=3140-4262 \mathrm{~ms}, \mathrm{TE}=100 \mathrm{~ms}$, slice thickness $=3 \mathrm{~mm}$; FLAIR: $\mathrm{TR}=8000 \mathrm{~ms}, \mathrm{TE}=90 \mathrm{~ms}$, slice thickness $=3 \mathrm{~mm} ; \mathrm{T} 2 * \mathrm{~W}: \mathrm{TR}=500, \mathrm{TE}=40 \mathrm{~ms}$, slice thickness $=3 \mathrm{~mm} ; \mathrm{T} 1 \mathrm{~W}$ : $\mathrm{TR}=582 \mathrm{~ms}, \mathrm{TE}=13 \mathrm{~ms}$, slice thickness $=3 \mathrm{~mm}$ ). Gadolinium (Dotarem $0.5 \mathrm{mmol} / \mathrm{mL}$, Dotarem-Guerbet Laboratories: Madrid, Spain) was used as contrast agent at a dose of $0.2 \mathrm{~mL} / \mathrm{kg}$ IV. DWI was not performed and cerebrospinal fluid was not collected in order to shorten the anesthetic time because the patient became hemodynamically unstable.

The MRI study showed an intra-axial lesion with well-defined margins and a triangle-shaped appearance on transverse images, located in the rostromedial portion of the right cerebellar hemisphere and the right side of the vermis. Compared to gray matter, the lesion was hyperintense on T2W, FLAIR and T2* images. On T1W images, the lesion was hypointense and showed no contrast enhancement. A 
mild mass effect that caused a midline shift toward the left was also observed. These MRI findings were consistent with an ischemic infarct in the territory of the right rostral cerebellar artery (Fig 1). Other less likely differential diagnoses included inflammatory/infectious encephalitis or infiltrative neoplasia such as lymphoma.

Thromboelastography was performed in order to assess hemostasis and it was unremarkable. Echocardiography was encouraged but rejected by the owner.

A serologic test for E cuniculi was also performed and it was positive (IgM 1:480; IgG 1:1280).

The patient was hospitalized for 3 days and fluid-therapy was started using crystalloid fluids (Ringer Lactate Braun 500 mL, Braun, Kronberg, Germany)) at a maintenance rate of $4 \mathrm{~mL} / \mathrm{kg} / \mathrm{h}$ IV, and she was placed in an oxygen cage in order to maintain brain perfusion and to avoid cerebral hypoxia. Assisted feeding was initiated with a commercial powdered preparation (Oxbow Critical Care, Oxbow Animal Health: NE). The patient showed significant improvement during hospitalization and was discharged 3 days later with fenbendazole at $20 \mathrm{mg} / \mathrm{kg} / \mathrm{PO}$ every 24 hours for 28 days (Panacur $100 \mathrm{mg} / \mathrm{mL}$, MSD Animal Health, Salamanca, España). Although the authors consider $E$ cuniculi unlikely to be the cause of the clinical signs, treatment was instituted because it is highly recommended when serological titers are elevated and indicate active infection. A gradual improvement in neurological signs was observed on weekly rechecks performed during the first 2 months. Five months later, the rabbit had no clinical signs of vestibular disease.

\section{Discussion}

Vestibular syndrome is the most common neurological abnormality in rabbits, and it has been traditionally classified as peripheral or central. To differentiate peripheral from central vestibular syndrome can be challenging, but sensitivity of the neurological localization rises up to $90 \%$ in small animals when it is performed accurately. ${ }^{6}$ Performance of an accurate neurological examination in pet rabbits is specially challenging given their prey nature and their tendency to remain still when they feel stressed or uneasy and even more when rabbits are rolling. ${ }^{1}$ In addition, differentiation of central and peripheral vestibular syndrome can also be difficult due to the high frequency of $E$ cuniculi central nervous system infections causing signs of peripheral disfunction. ${ }^{1,7}$

Paradoxycal vestibular syndrome has never been reported in this species regardless of the underlying cause and it is not frequent either in small animals. However, in the cases described, inflammatory disease or neoplasia are the most frequent final diagnoses. ${ }^{8}$

The most common cause of central vestibular disease in rabbits is granulomatous encephalitis caused by the microsporidian $E$ cuniculi and it is frequently transmitted through ingestion of spores. The way the patient of this report was infected is unknown, although the microsporidian could have been latent since adoption and reactivate later on. Even if there is a high percentage of rabbits seropositive to the parasite (50\%-70\%), ${ }^{7}$ it is frequent that the infection is chronic and asymptomatic, ${ }^{1}$ so the fact that both serological titers were elevated did not necessarily indicate that encephalitozoonosis was the cause of the clinical signs. In addition, there is no correlation between the severity of the E cuniculi-induced histopathological lesions in the brain and neurological signs. ${ }^{9}$

Other reported causes of central vestibular syndrome in rabbits include intracranial neoplasms such as lymphoma or other inflammatory encephalopathies, but vascular lesions have not been identified as primary causes of vestibular syndrome in rabbits and the few mentions on vascular lesions causing neurological signs are secondary to hypoxia. ${ }^{2}$ This lack of evidence of ischemic infarcts in rabbits can be due to the fact that brain MRI is not routinely performed in patients with vestibular disease and the diagnosis usually relies on more basic testing like radiographs of the tympanic bullae and serology for $E$ cuniculi infection. It is widely known that MRI is more expensive and requires long times of anesthesia, sometimes leading caregivers to choose a more conservative diagnostic approach that might be inconclusive. It is currently unknown whether granulomatous encephalitis caused by E cuniculi can predispose to ischemic infarcts in rabbits.

Ischemic infarcts are more frequently included in the differential diagnosis of dogs with acute, nonprogressive central vestibular signs, paradoxical or not. ${ }^{10}$ Predisposing factors in canines include endocrinopathies (Cushing's syndrome, diabetes mellitus, and hypothyroidism) or chronic renal failure. However, an underlying cause cannot be found in up to 50\% of dogs with brain infarction. ${ }^{11}$ The tests performed in the rabbit presented here did not show any results compatible with endocrinopathies

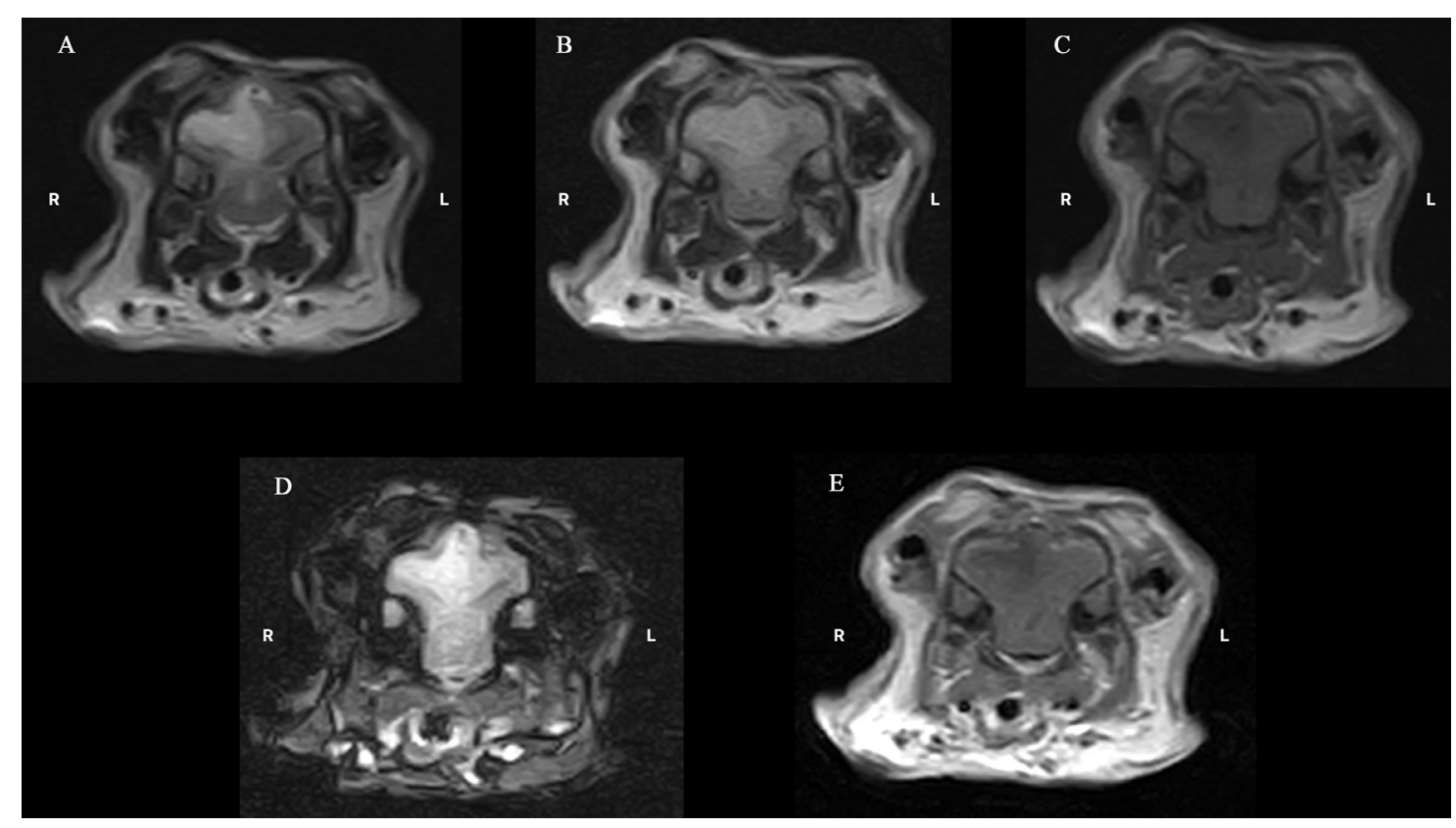

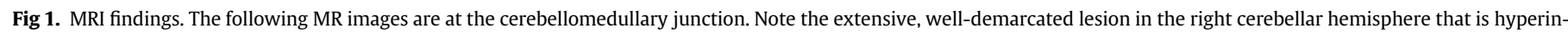
tense on T2W (A), FLAIR (B), and T2* (D) images, and hypointense on T1W (C), without contrast enhancement (E). 
and thromboelastography was normal, so unfortunately no factors that could lead to a prothrombotic status could be identified.

The rabbit's cerebellar vascular supply is carried by 2 sets of arteries. The caudal cerebellar artery (right and left), which originates from the basilar artery, and the rostral cerebellar artery (right and left) that has its origin in the caudolateral aspect of the circle of Willis. In a small percentage of animals, the right internal carotid artery sends a vessel at the level of the cerebellar peduncles that anastomoses with the terminal portion of the basilar artery, creating the right rostral cerebellar artery. The rostral cerebellar artery is usually double on the right side and single on the left. In cases in which it is double, it vascularizes almost the entire cerebellum. The caudal cerebellar artery is often single and can be found at the trapezoid body generating a lateral branch of the basilar artery. It can also be double or triple in some cases. ${ }^{12}$ Therefore, and despite some differences, the cerebellar vascular supply in rabbits is similar to that in canines. In dogs, most ischemic cerebellar infarcts occur in the rostral cerebellar artery territory. ${ }^{13}$ Involvement of the caudal cerebellar artery has been rarely reported in dogs. ${ }^{14}$

Ischemic strokes appear hyperintense on T2W and hypo-/isointense on T1W images. ${ }^{15}$ On FLAIR sequences their appearance is similar to that on $\mathrm{T} 2$, and on $\mathrm{T} 2 *$ they can be mildly hyperintense or isointense. ${ }^{15}$ Contrast enhancement may or may not be seen. ${ }^{15}$ Diffusion-weighted imaging in the acute stages of the disease shows a focal area of increased signal that corresponds with a hypointense area on the apparent diffusion coefficient map, caused by a restriction in water diffusion. ${ }^{16}$ In addition, most strokes appear wedge or rectangular shaped, although other patterns like ovoid or pinpoint have also been reported. ${ }^{16}$ Eventhough diffusion-weighted imaging was not performed for the reasons aforementioned, the MRI study was consistent with an infarct, showing features similar in shape, intensity, and localization to those in dogs.

Prognosis in canines with strokes is considered fair to good with a survival rate of approximately $60 \% .{ }^{11}$ The clinical progression of the rabbit reported here, despite treatment for encephalitozoon, resembles that in dogs with brain infarction.

\section{Conclusions}

To the authors knowledge this is the first case report of a paradoxical vestibular syndrome in a rabbit pet, presumptively caused by a cerebellar infarct. It may be concluded that though rare, ischemic infarcts should be included in the differential diagnosis of central vestibular syndrome in rabbits. MRI is essential for diagnosis and should be included in the diagnostic protocol of central neurological disease in rabbits.

\section{Authors' Contributions}

RG was an assistant clinician and wrote the article. VS was the primary clinician of the case. RN performed the magnetic resonance, informed the images and reviewed the article. SA supervised the case and reviewed the article. JM supervised the case and reviewed the article. $\mathrm{CF}$ reviewed the article.

\section{References}

1. Künzel F, Gruber A, Tichy A, Edelhofer R, Nell B, Hassan J, Leschnik M, Thalhammer JG, Joachim A. Clinical symptoms and diagnosis of encephalitozoonosis in pet rabbits. Vet Parasitol 151:115-124, 2008

2. Gruber A, Pakozdy A, Weissenböck H, Csokai J, Künzel F. A retrospective study of neurological disease in 118 Rabbits. J Comp Pathol 140:31-37, 2009

3. Cheng L, Guo P, wei Liao Y, liang Zhang H, ting Li H, Yuan X. Pathophysiological changes in the cerebellum and brain stem in a rabbit model after superior petrosal vein sacrifice. Biosci Rep 38:1-9, 2018

4. Jahan R, Villablanca JP, Harris RJ, et al. Selective middle cerebral artery occlusion in the rabbit: technique and characterization with pathologic findings and multimodal MRI. J Neurosci Methods 313:6-12, 2019

5. Meyer DM, Chen YM, Zivin JA. Dose-finding study of phototherapy on stroke outcome in a rabbit model of ischemic stroke. Neurosci Lett 630:254-258, 2016

6. Bongartz U, Nessler J, Maiolini A, Stein VM, Tipold A, Bathen-Nöthen A. Vestibular disease in dogs: association between neurological examination, MRI lesion localisation and outcome. J Small Anim Pract 61:57-63, 2020

7. Jass A, Matiasek K, Henke J, Küchenhoff H, Hartmann K, Fischer A. Analysis of cerebrospinal fluid in healthy rabbits and rabbits with clinically suspected encephalitozoonosis. Vet Rec 162:618-622, 2008

8. de Lahunta A, Glass E, Kent M. Veterinary neuroanatomy and clinical neurology. 4th ed. St Louis: Elsevier; 2015. p. 346-347

9. Csokai J, Gruber A, Künzel F, Tichy A, Joachim A. Encephalitozoonosis in pet rabbits (Oryctolagus cuniculus): pathohistological findings in animals with latent infection versus clinical manifestation. Parasitol Res 104:629-635, 2009

10. Thomsen B, Garosi L, Skerritt G, et al. Neurological signs in 23 dogs with suspected rostral cerebellar ischaemic stroke. Acta Vet Scand 58:1-9, 2016

11. Garosi L, McConnell JF, Platt SR, et al. Results of diagnostic investigations and longterm outcome of 33 dogs with brain infarction (2000-2004). J Vet Intern Med 19:725-731, 2005

12. de Souza F, Campos R. A systematic study of the brain base arteries in the rabbit (oryctolagus cuniculus). Pesqui Vet Brasil 33:796-806, 2013

13. Garosi L, McConnell JF, Platt SR, et al. Clinical and topographic magnetic resonance characteristics of suspected brain infarction in 40 dogs. J Vet Intern Med 20:311321,2006

14. Negrin A, Gaitero L, Añor S. Presumptive caudal cerebellar artery infarct in a dog: clinical and MRI findings. J Small Anim Pract 50:615-618, 2009

15. Mai W. Diagnostic MRI in dogs and cats. 1st ed. Boca Raton: Taylor \& Francis Group; 2018. p. 259-279

16. McConnell JF, Garosi L, Platt SR. Magnetic resonance imaging findings of presumed cerebellar cerebrovascular accident in twelve dogs. Vet Radiol Ultrasound 46:1-10, 2005 